\title{
Un RÉQUiEM PARA la Regla de KAISER (EIGEN > 1) EN LA RETENCIÓN DEL NÚMERO DE FACTORES ${ }^{\star}$
}

\author{
Jhonatan S. Navarro Loli $\star$ \\ Cesar A. Merino Soto ${ }^{\star \star}$
}

\section{Palabras clave: Análisis Factorial Exploratorio; Análisis Paralelo; regla Kaiser.}

El presente trabajo describe una propuesta relevante para tomar una decisión acertada en la determinación del número de factores para retener en un análisis factorial exploratorio. El análisis factorial es una técnica de análisis multivariado que es utilizado en ciencias sociales y ciencias de la salud, y de él se derivan dos enfoques: el análisis factorial confirmatorio y el análisis factorial exploratorio (AFE), siendo el paso más crítico e importante en este último la extracción del número de factores. En el proceso de determinar el número de factores a retener en un AFE, y dependiendo de las condiciones y técnicas que se utilice, puede ocurrir una sobreestimación o en el peor de los casos una subestimación del número de factores. Hay varias técnicas para la extracción del numero de factores, entre ellas el Análisis Paralelo (AP) y la regla Kaiser (eigen > 1) (K1), siendo esta última la más popular y consecuentemente la más utilizada. Sin embargo, el uso de esta última presenta serios problemas metodológicos y limitaciones que aparentemente no hacen elegible esta regla. Como evidencia de estos problemas tenemos los numerosos estudios de simulación que indican que el uso de la regla Kaiser tiende a sobreestimar el número de factores en $1 / 3 ; 1 / 5$ y $1 / 6$ el número real de factores, además su precisión empeora a medida que hay un aumento del número de variables, cuando el tamaño de la muestra aumenta, la magnitud de las comunalidades y la proporción de sujetos por variable son bajas. Esto lleva a los investigadores a concluir que no hay la suficiente evidencia empírica que respalde la continua aplicación contemporánea de la regla Kaiser. En contraste a esta regla tenemos al Análisis Paralelo, debido a que, en los estudios de simulación realizados en los que comparan la precisión del Análisis Paralelo con la regla Kaiser, es la técnica que tiende a estimar de manera más precisa (entre un $90 \%$ y 95\%) el número de factores; por lo tanto es la técnica que cuenta con mayor respaldo científico y consecuentemente la que más se recomienda. Tomando en cuenta las evidencias que existen, la utilización de la regla Kaiser ocasiona serios problemas en la interpretatibilidad de los factores, afectando seriamente a la calidad psicométrica de un test psicológico y de las evidencias de validez de la estructura interna, lo cual trae como consecuencia directa que las inferencias que el

\footnotetext{
^ Ponencia presentada en el I Congreso Peruano de Estudiantes de Psicología y II Congreso Nacional de Estudiantes de Psicología Aplicada, del 4 al 6 de Agosto, 2010, Chiclayo, Perú.

$\star \star$ Estudiante de Psicología en la Facultad de Psicología de la Universidad Nacional Federico Villareal. E-mail: jhonatan_navarro1602@yahoo.es

$\star \star \star$ Psicólogo, Magister en Psicología Educativa, investigador del Instituto de Investigación en Psicología (Universidad de San Martín de Porres y en la Universidad Cientifica del Sur) y profesor en la Universidad Científica del Sur y Universidad de San Martín de Porres.

E-mail: sikayax@yahoo.com.ar
} 
investigador pueda adoptar desde las puntuaciones del test se vean afectadas. Se discute el papel de los programas informáticos de estadística populares respecto la inclusión de este procedimiento, así como recomendaciones para una práctica efectiva en la retención del número de factores. 